\title{
Time Delay in the Recoiling Valence Photoemission of Ar Endohedrally Confined in $\mathrm{C}_{60}$
}

\author{
Gopal Dixit, ${ }^{1,2, *}$ Himadri S. Chakraborty, ${ }^{3, \dagger}$ and Mohamed El-Amine Madjet ${ }^{1,4, \ddagger}$ \\ ${ }^{1}$ Center for Free-Electron Laser Science, DESY, Notkestrasse 85, 22607 Hamburg, Germany \\ ${ }^{2}$ Max Born Institute, Max-Born-Strasse 2A, 12489 Berlin, Germany \\ ${ }^{3}$ Department of Natural Sciences, Center for Innovation and Entrepreneurship, Northwest Missouri State University, \\ Maryville, Missouri 64468, USA \\ ${ }^{4}$ Qatar Energy and Environment Research Institute (QEERI), Qatar Foundation, Doha 5825, Qatar
}

(Received 7 July 2013; published 12 November 2013)

\begin{abstract}
The effects of confinement and electron correlations on the relative time delay between the $3 s$ and $3 p$ photoemissions of $\mathrm{Ar}$ confined endohedrally in $\mathrm{C}_{60}$ are investigated using the time-dependent local density approximation - a method that is also found to mostly agree with recent time delay measurements between the $3 s$ and $3 p$ subshells in atomic Ar. At energies in the neighborhood of $3 p$ Cooper minimum, correlations with $\mathrm{C}_{60}$ electrons are found to induce opposite temporal effects in the emission of $\operatorname{Ar} 3 p$ hybridized symmetrically versus that of $\operatorname{Ar} 3 p$ hybridized antisymmetrically with $\mathrm{C}_{60}$. A recoil-type interaction model mediated by the confinement is found to best describe the phenomenon.
\end{abstract}

With the tremendous advancement in technology for generating attosecond (as) isolated pulses as well as attosecond pulse trains, it becomes possible to study fundamental phenomena of light-matter interaction with unprecedented precision on an as time scale [1-3]. In particular, the relative time delay between the photoelectrons from different subshells on as time scale, a subject of intense recent activities, is expected to probe important aspects of electron correlations that predominantly influence the photoelectron. Pump-probe experiments have been performed to measure the relative delay in the photoemission processes, where extreme ultraviolet (XUV) pulses are used to remove an electron from a particular subshell and subsequently a weak infrared (IR) pulse accesses the temporal information of the emission event [4].

Streaking measurements were carried out to probe photoemission from the valence and the conduction band in single-crystalline magnesium [5] and tungsten [6]. A streaking technique was also employed to measure the relative delay of approximately $21 \pm 5$ as between the $2 s$ and $2 p$ subshells of atomic Ne at $106 \mathrm{eV}$ photon energy [7]. Despite several theoretical attempts [8-14] to explain this measured delay in $\mathrm{Ne}$, only about a half of the delay could be reproduced, keeping the time delay in Ne photoemissions still an open problem. Recently, the relative delay between the $3 s$ and $3 p$ subshells in Ar is measured at three photon energies by an interferometric technique using attosecond pulses $[15,16]$. Theoretical methods (e.g., time-dependent nonperturbative method [8], diagrammatic many-body perturbation theory [13], random phase approximation with exchange (RPAE) [14,16], and multiconfigurational Hartree-Fock (MCHF) [17]) have been employed to investigate this relative delay in Ar, although agreements between theory and experiment are rather inconclusive. A ubiquitous understanding in all these studies is the dominant influence of electron correlations to determine the time behavior of outgoing electrons. Thus, it is fair to expect that the process near a Cooper minimum or a resonance will be particularly nuanced.

It is therefore of spontaneous interest to extend the study to test the effect of correlations on the temporal photoresponse of atoms in material confinements. A brilliant natural laboratory for such is an atom endohedrally captured in a fullerene shell; see Fig. 1 which envisions the process. There are two compelling reasons for this choice: (i) such materials are highly stable, have low-cost sustenance at room temperature, and are enjoying a rapid improvement in their synthesis techniques [18] and (ii) effects of correlations of the central atom with the cage electrons have been predicted to spectacularly influence the atomic valence photoionization [19]. In this Letter, by considering $\mathrm{Ar} @ \mathrm{C}_{60}$, we show that a confinement-induced correlation effect of $\mathrm{C}_{60}$ at energies surrounding the $\operatorname{Ar} 3 p$ Cooper minimum produces a faster and a slower emission of the Ar $3 p$ electrons hybridized, respectively, in a symmetric and an antisymmetric mode with a near-degenerate $\mathrm{C}_{60}$ orbital.

Time-dependent local density approximation (TDLDA), with Leeuwen and Baerends (LB) exchange-correlation functional to produce accurate asymptotic behavior [20] of ground and continuum electrons is employed to calculate the dynamical response of the system to the external electromagnetic field. To demonstrate the accuracy of the method for an isolated atom, the total photoionization cross section and the partial $3 s$ and $3 p$ cross sections of Ar are presented in Fig. 2(a) and compared with available experiments [21,22]. As seen, our TDLDA total and $3 s$ cross sections are in excellent agreement with experimental results and the positions of the $3 s$ and $3 p$ Cooper minima at, respectively, 42 and $48 \mathrm{eV}$ are well reproduced. The dominance of $3 p$ contribution over $3 s$ in this energy range [Fig. 2(a)] also automatically implies the accuracy of our TDLDA $3 p$ result. 

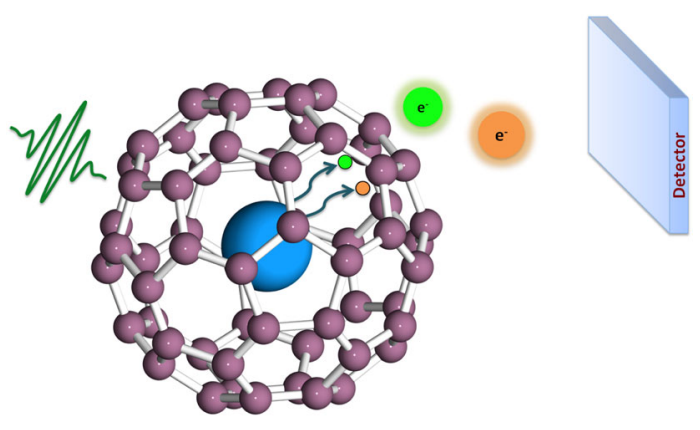

FIG. 1 (color online). Schematic for probing the effects of correlations from the confinement on the relative time delay in the emission of an atom encaged endohedrally inside $\mathrm{C}_{60}$.

The absolute time delay in Ar pump-probe photoemission contains two contributions: one due to the absorption of XUV photon and the other due to the probe pulse. Owing to the weak probe pulse, the probe-assisted delay contributions can be estimated [13] as a function of the kinetic energy of electrons from different Ar subshells. This allowed for the evaluation of the relative delay essentially due to the XUV pump photon in recent measurements $[15,16]$. This delay therefore connects to the energy derivative of the quantum phase of complex

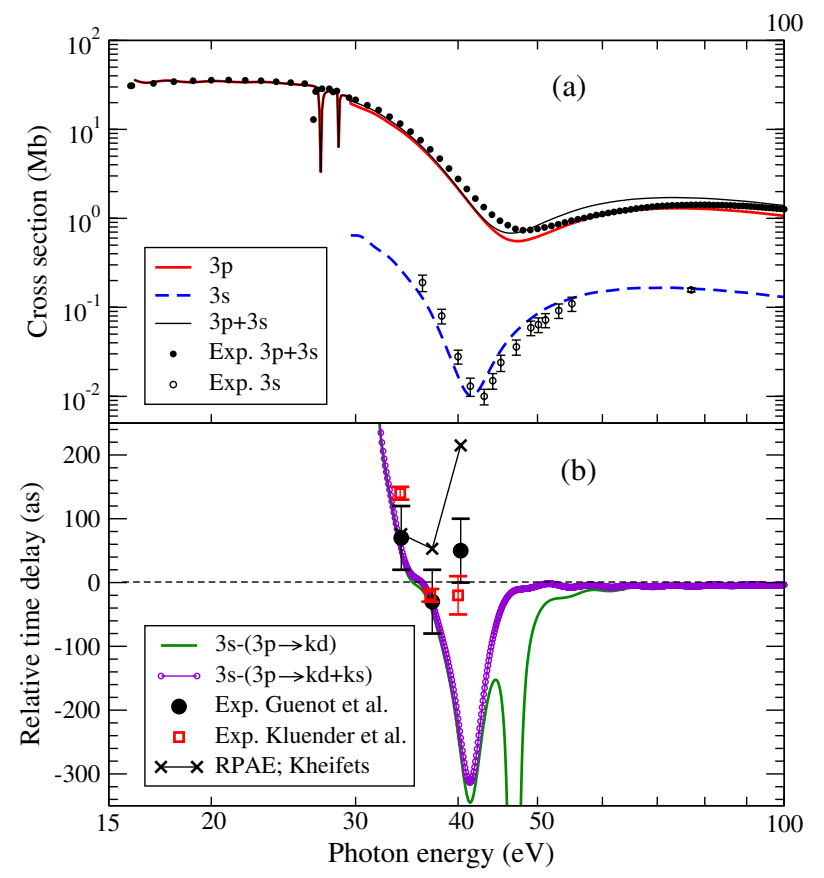

FIG. 2 (color online). Top: TDLDA $3 p, 3 s$ and total photoionization cross sections for atomic $\mathrm{Ar}$ are compared with experiments for $3 s$ [21] and total [22]. For $3 s$ the computed cross section is scaled to reproduce the measurement at the Cooper minimum. Bottom: The relative TDLDA time delay between $3 s$ and $3 p$ of Ar and its comparison with measurements (solid black circles, Ref. [16]; open red squares, Ref. [15]). RPAE results [14] at three experimental energies are also cited. photoionization amplitude [23] - the Wigner-Smith time delay [24-26]. Several methods [11,12,27,28] have been utilized to extract the Wigner-Smith time delay directly from the measurements.

The photoionization amplitude from an initial bound state $\left(n_{i} l_{i}\right)$ to a final continuum state $(k l)$ can be expressed as

$$
\begin{aligned}
f(\hat{\mathbf{k}})= & (8 \pi)^{3 / 2} \sum_{\substack{l=l_{i} \pm 1 \\
m=m_{i}}}(-i)^{l} e^{i \eta_{l}(\hat{\mathbf{k}})} Y_{l m}^{*}(\hat{\mathbf{k}})\left\langle\phi_{k l}\|r+\delta V\| \phi_{n_{i} l_{i}}\right\rangle \\
& \times \sqrt{(2 l+1)\left(2 l_{i}+1\right)}\left(\begin{array}{ccc}
l & 1 & l_{i} \\
0 & 0 & 0
\end{array}\right)\left(\begin{array}{ccc}
l & 1 & l_{i} \\
-m & 0 & m_{i}
\end{array}\right) .
\end{aligned}
$$

Here, $\delta V$ is the complex induced potential which embodies TDLDA many-body correlations. The phase $\eta_{l}$ includes contributions from both the short-range and Coulomb potentials, whereas the phase of the complex matrix element in Eq. (1) is the correlation phase. For Ar, the correlation near Cooper minima primarily arises from the coupling of $3 p$ with $3 s$ channels. The total phase is the sum of these three contributions. The time delay profile is computed by differentiating the TDLDA total phase in energy.

Our TDLDA relative Wigner-Smith delay between Ar $3 s$ and $3 p, \tau_{3 s}-\tau_{3 p}$, is compared with the experimental data of Guénot et al. [16] and of Klünder et al. [15] in Fig. 2(b). As seen, the relative delay is strongly energy dependent. Note that the TDLDA results are in excellent agreement with both sets of experimental results at 34.1 and $37.2 \mathrm{eV}$. The third measurement at $40.3 \mathrm{eV}$, which is in the vicinity of the $3 s$ Cooper minimum, is negative in Ref. [15] in contrast to its positive value in Ref. [16]. Note that our result captures the correct sign as in Klünder et al. at $40.3 \mathrm{eV}$. In general, $3 p \rightarrow k d$ photochannel is dominant over $3 p \rightarrow k s$ at most energies. Close to the $3 p$ Cooper minimum, however, $3 p \rightarrow k d$ begins to rapidly decrease to its minimum value, enabling $3 p \rightarrow k s$ to significantly contribute to the net $3 p$ delay. The $s$ - and $d$-wave emissions have different angular distributions, but their Wigner delays are independent of emission directions. Thus, assuming that all $3 p$ photoelectrons are detected (integration over solid angle), the net $3 p$ delay must be a statistical combination, that is, the sum of the delays weighted by the channel's individual cross section branching ratios. As illustrated in Fig. 2(b), upon including $3 p \rightarrow k s$ along with $3 p \rightarrow k d$ (purple curve) this way, the shape of the TDLDA delay strikingly alters near the $3 p$ Cooper minimum. We stress that the delay near a Cooper minimum needs to be addressed with great care which can reveal new physics, as shown below for an endohedrally confined Ar atom.

We also include recent RPAE results [14] for three experimental energies in Fig. 2(b). As seen, RPAE and 
experiments match only at $34.1 \mathrm{eV}$. The superior performance of TDLDA in explaining the measurements is thus evident. While both TDLDA and RPA are many-body linear response theories, they have significant differences in the details, particularly in treating electron correlations [29]. Variants of the Kohn-Sham LDA + LB scheme were successfully utilized to describe attosecond strong-field phenomena [30-34], underscoring the reliability of many-body correlations that TDLDA characteristically offers.

This success of the TDLDA method for free Ar encouraged us to use the approach to investigate the delay in an $\mathrm{Ar}$ atom endohedrally sequestered in $\mathrm{C}_{60}$. The jellium model is employed for computing the relative delay [35]. This model enjoyed earlier successes in codiscovering with experimentalists a high-energy plasmon resonance [36], interpreting the energy-dependent oscillations in $\mathrm{C}_{60}$ valence photo intensities [37], and predicting giant enhancements in the confined atom's photoresponse from the coupling with $\mathrm{C}_{60}$ plasmons [19]. Significant ground state hybridization of $\operatorname{Ar} 3 p$ is found to occur with the $\mathrm{C}_{60} 3 p$ orbital, resulting in $3 p\left[\mathrm{Ar}+\mathrm{C}_{60}\right]$ and $3 p\left[\mathrm{Ar}-\mathrm{C}_{60}\right]$ from, respectively, the symmetric and antisymmetric wave function mixing. These are spherical analogs of bonding and antibonding states in molecules or dimers. Such atomfullerene hybridization was predicted before [38] and detected in the photoemission experiment on multilayers of $\mathrm{Ar} @ \mathrm{C}_{60}$ [39]. In fact, the hybridization gap of $1.5 \mathrm{eV}$ between $3 p\left[\mathrm{Ar}+\mathrm{C}_{60}\right]$ and $3 p\left[\mathrm{Ar}-\mathrm{C}_{60}\right]$ in our calculation is in good agreement with the measured value of $1.6 \pm$ $0.2 \mathrm{eV}$ [39].

The TDLDA Wigner-Smith phases for relevant ionization channels for confined and free Ar are presented in Fig. 3. We use the symbol "@” to denote states belonging to the confined Ar. The narrow resonance spikes below $40 \mathrm{eV}$ are due to single electron Rydberg-type excitations in $\mathrm{C}_{60}$. This energy zone also includes the $\mathrm{C}_{60}$ plasmon resonances, although their effects are suppressed by the Coulomb phase that dominates the extended region above ionization thresholds. We note that the Ar $3 s$ Cooper minimum shifts slightly lower in energy to $36.5 \mathrm{eV}$ from the confinement, but the confinement moves the two $3 p$ minima, each in the bonding and antibonding channels, somewhat higher in energy. What is rather dramatic in Fig. 3 is that the quantum phase corresponding to $3 p\left[\mathrm{Ar}+\mathrm{C}_{60}\right] \rightarrow \mathrm{kd} @$ (thick solid black) makes a downward $\pi$ phase shift, whereas the phase associated with $3 p\left[\mathrm{Ar}-\mathrm{C}_{60}\right] \rightarrow k d @$ (thick solid red) suffers an upward $2 \pi$ phase shift at their respective Cooper minimum. Further note that both these contributions together yield a net phase that shifts up by $\pi$ as in the case of free-Ar $3 p \rightarrow k d$ channel (dashed black curve in Fig. 3) at its Cooper minimum.

This contrasting phase behavior between hybrid $3 p$ emissions is likely the effect of symmetric and antisymmetric wave function shapes on the matrix elements through dynamical correlations. Using the wellknown Fano scheme of perturbative interchannel coupling [40], the lead contribution to the matrix element $\langle\delta V\rangle$ [Eq. (1)] is [41]

$$
\langle\delta V\rangle_{\alpha}(E)=\sum_{\beta} \int d E^{\prime} \frac{\left\langle\Psi_{\beta}\left(E^{\prime}\right)\left|\frac{1}{\left|\mathbf{r}_{\alpha}-\mathbf{r}_{\beta}\right|}\right| \Psi_{\alpha}(E)\right\rangle}{E-E^{\prime}}\langle z\rangle_{\beta}\left(E^{\prime}\right),
$$

where $\alpha$ denotes each of the $3 p\left[\mathrm{Ar} \pm \mathrm{C}_{60}\right] \rightarrow k d @$ channels. $\Psi$ are channel wave functions that involve both bound (hole) and continuum (photoelectron) states, and $\langle z\rangle_{\beta}$ is the single channel matrix element of each perturbing channel $\beta$. Thus, the summation over channels incorporates bound states as the hole states. Two points can be noted: First, $\langle\delta V\rangle$ dominates near the Cooper minimum of a channel $\alpha$, since the "unperturbed" $\langle z\rangle_{\alpha}$ is already small at these energies; second, $\langle\delta V\rangle$ depends on the coupling matrix element in the numerator of Eq. (2) that involves overlaps between the bound state of a $\alpha$ channel with that in a perturbing $\beta$ channel. These overlaps are critical, since $3 p\left[\mathrm{Ar}+\mathrm{C}_{60}\right]$ wave function has a structure completely opposite to that of $3 p\left[\mathrm{Ar}-\mathrm{C}_{60}\right]$ over the $\mathrm{C}_{60}$ shell region, where each of them strongly overlaps with a host of $\mathrm{C}_{60}$ wave functions to build correlations. These opposing modes of overlap from one hybrid to another flip the phase modification direction between two hybrid $3 p$ emissions around a respective Cooper minimum, as seen in Fig. 3.

Depending on the upward (downward) shift in the quantum phase, the resulting photoelectron exhibits positive (negative) time delay and hence emerges slower (faster) from the ionization region. This is evident in Fig. 4(a), which

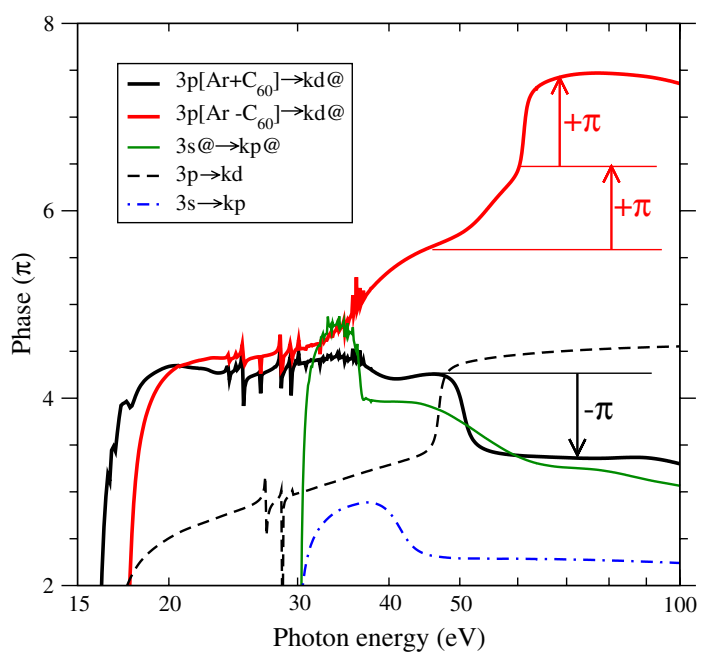

FIG. 3 (color online). TDLDA quantum phases for ionization via $d$-waves from bonding $3 p\left[\mathrm{Ar}+\mathrm{C}_{60}\right]$ and antibonding $3 p\left[\mathrm{Ar}-\mathrm{C}_{60}\right]$ levels and via $p$-wave from Ar $3 s @$ are compared with their counterparts in free Ar. 
features various absolute delays: Channels $3 p\left[\mathrm{Ar}+\mathrm{C}_{60}\right] \rightarrow$ $k d @$ and $3 p\left[\mathrm{Ar}-\mathrm{C}_{60}\right] \rightarrow k d @$ exhibit, respectively, a fast and a slow emission over relatively narrow ranges about their Cooper minima. Note that the peak delay of the antibonding electron is approximately double to the peak advancement (negative delay) of the bonding electron. The delay profile becomes softer and broader in energy by including the contribution from the $s$-wave, but the general trend of a rapid and a slow ejection, respectively, in the bonding-antibonding channels survives.

The conservation of the quantum phase, i.e., the net phase shift of $\pi$ in the upward direction (as in the free Ar) for $3 p$ in $\mathrm{Ar} @ \mathrm{C}_{60}$ can be understood in the language of a collision-type interaction between two hybrid $3 p$ electrons. The phase behaves like the linear momentum in a two-body collision, which is a conserved quantity. Its energy derivative, i.e., the time delay, can be thought to be commensurate with the collision force, the time derivative of the momentum, since time and energy are conjugate variables. This implies that if one hybrid electron goes through an advanced emission, the other hybrid must delay or time-recoil appropriately to keep the net delay roughly close to the delay of free Ar. Of course, here the process is underpinned by the orbital mixing. Therefore, the phenomenon can be pictured as the photo-liberation of two recoiling electrons in the temporal domain from the

100

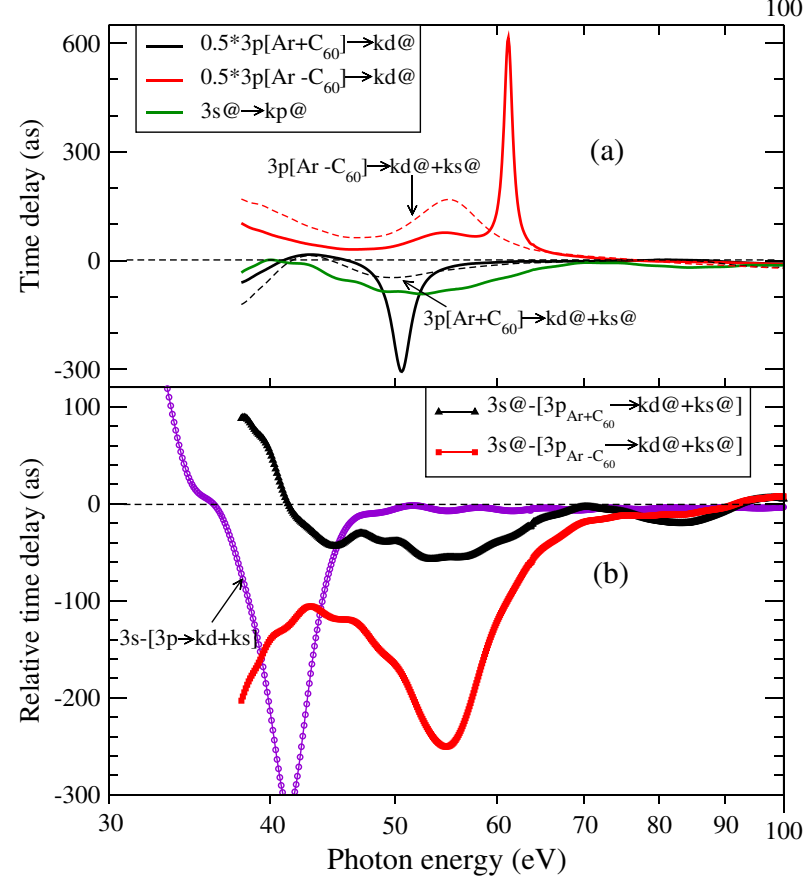

FIG. 4 (color online). Top: Absolute time delay for ionizations in 3p $\left[\mathrm{Ar} \pm \mathrm{C}_{60}\right] \rightarrow k d @$ and $3 s @ \rightarrow k p @$ channels. For the two hybrid channels, results modified by incorporating $s$-wave delays are also shown. Bottom: Relative delays $\tau_{3 s @-}$ $\tau_{3 p\left[\mathrm{Ar} \pm \mathrm{C}_{60}\right]}$, including the $s$-wave contributions; $\tau_{3 s}-\tau_{3 p}$ of free $\mathrm{Ar}$ is also shown for comparison. atom-fullerene hybridization. Hence, it is also likely to exist in the ionization of molecules, nanodimers, and fullerene onions that support hybrid electrons.

The time delays in the photoionization of $3 p$ hybrids (with $s$-wave contribution included) relative to $3 s @$, $\tau_{3 s @}-\tau_{3 p\left[\mathrm{Ar}^{ \pm} \mathrm{C}_{60}\right]}$ are presented in Fig. 4(b). One notes in Fig. 4(a) that 3s@ $\rightarrow k p @$ produces an absolute delay profile, which is negative for most energies and, on average, comparable to the absolute delay in $3 p\left[\mathrm{Ar}-\mathrm{C}_{60}\right] \rightarrow$ $k d @+k s @$. Consequently, their (fast) emergence at about similar speeds keeps their relative delay close to $\tau=$ 0 , but with a bias toward negative values. On the other hand, for the 3p $\left[\mathrm{Ar}+\mathrm{C}_{60}\right] \rightarrow k d @+k s @$ channel, the relative delay remains mostly strongly negative. However, the rich structures in the delay profiles emphasize that the Cooper minimum regions are particularly attractive for time delay studies.

The $3 p$ bonding-antibonding gap of $1.5 \mathrm{eV}$ requires the energy of the probe pulse to be smaller than this gap. Otherwise, the sideband of one level will begin to overlap with the harmonics of the other. Also, by varying the polarization angle between XUV and IR pulses, one can potentially probe both independent contributions, i.e., the relative delay between $3 s$ orbital and $3 p$ bondingantibonding orbital, i.e., by extending the standard RABBIT method [42], where the polarization of XUV pulse is the same as the IR pulse. Therefore, techniques based on interferometry, such as RABBIT and PROOF [43], have potentials to probe the relative delay between $3 p$ bonding-antibonding and $3 s$ electrons. One may also perform the streaking experiments using IR as well as THz pulses for accessing the delay. We suggest that future experiments be performed on the time delay in $\mathrm{Ar}$ and $\mathrm{Ar} @ \mathrm{C}_{60}$ over broader photon energy ranges including the $3 p$ Cooper minimum to unravel new physics from confinement and correlations.

In conclusion, our TDLDA relative Wigner-Smith time delay between $3 s$ and $3 p$ subshells in free Ar is in excellent agreement with the measured delay except near the $3 \mathrm{~s}$ Cooper minimum, where, however, the TDLDA is consistent with the sign of one set of measurements. In the case of confined Ar, due to the electron correlation, the delays of the $3 p$ bonding and $3 p$ antibonding emissions are governed by a recoil-type emission in the time domain mediated by the host $\mathrm{C}_{60}$. It is found that the emission from the $3 s @$ level is slightly faster than the emission from the $3 p$ bonding level but is substantially faster, by 100 as and above, than the emission from the $3 p$ antibonding level. We further demonstrate that the delay of $\operatorname{Ar} 3 p$ electron, free or confined, leads to significant modifications in the vicinity of the Cooper minimum by including the $s$-wave photochannel.

G. D. acknowledges Misha Ivanov, Tim Laarmann, and Oliver Mücke for useful discussions. The research is supported by the NSF, USA. 
*dixit@mbi-berlin.de

†himadri@nwmissouri.edu

†mmadjet@qf.org.qa

[1] M. Hentschel, R. Kienberger, C. Spielmann, G. A. Reider, N. Milosevic, T. Brabec, P. Corkum, U. Heinzmann, M. Drescher, and F. Krausz, Nature (London) 414, 509 (2001).

[2] E. Goulielmakis, M. Schultze, M. Hofstetter, V.S. Yakovlev, J. Gagnon, M. Uiberacker, A. L. Aquila, E. M. Gullikson, D. T. Attwood, R. Kienberger, F. Krausz, and U. Kleineberg, Science 320, 1614 (2008).

[3] F. Krausz and M. Ivanov, Rev. Mod. Phys. 81, 163 (2009).

[4] R. Pazourek, S. Nagele, and J. Burgdörfer, Faraday Discuss. 163, 353 (2013).

[5] S. Neppl, R. Ernstorfer, E. M. Bothschafter, A. L. Cavalieri, D. Menzel, J. V. Barth, F. Krausz, R. Kienberger, and P. Feulner, Phys. Rev. Lett. 109, 87401 (2012).

[6] A. L. Cavalieri, N. Müller, T. Uphues, V.S. Yakovlev, A. Baltuska, B. Horvath, B. Schmidt, L. Blümel, R. Holzwarth, S. Hendel, M. Drescher, U. Kleineberg, P. M. Echenique, R. Kienberger, F. Krausz, and U. Heinzmann, Nature (London) 449, 1029 (2007).

[7] M. Schultze et al., Science 328, 1658 (2010).

[8] J. Mauritsson, M. B. Gaarde, and K. J. Schafer, Phys. Rev. A 72, 013401 (2005).

[9] A. S. Kheifets and I. A. Ivanov, Phys. Rev. Lett. 105, 233002 (2010).

[10] L. R. Moore, M. A. Lysaght, J. S. Parker, H. W. van der Hart, and K.T. Taylor, Phys. Rev. A 84, 061404 (2011).

[11] M. Ivanov and O. Smirnova, Phys. Rev. Lett. 107, 213605 (2011).

[12] S. Nagele, R. Pazourek, J. Feist, and J. Burgdörfer, Phys. Rev. A 85, 033401 (2012).

[13] J. M. Dahlström, T. Carette, and E. Lindroth, Phys. Rev. A 86, 061402 (2012).

[14] A. S. Kheifets, Phys. Rev. A 87, 063404 (2013).

[15] K. Klünder, J. M. Dahlström, M. Gisselbrecht, T. Fordell, M. Swoboda, D. Guénot, P. Johnsson, J. Caillat, J. Mauritsson, A. Maquet, R. Taïeb, and A. L'Huillier, Phys. Rev. Lett. 106, 143002 (2011).

[16] D. Guénot, K. Klünder, C. L. Arnold, D. Kroon, J. M. Dahlström, M. Miranda, T. Fordell, M. Gisselbrecht, P. Johnsson, J. Mauritsson, E. Lindroth, A. Maquet, R. Tä̈eb, A. L'Huillier, and A. S. Kheifets, Phys. Rev. A 85, 053424 (2012).

[17] T. Carette, J. M. Dahlström, L. Argenti, and E. Lindroth, Phys. Rev. A 87, 023420 (2013).

[18] A. A. Popov, S. Yang, and L. Dunsch, Chem. Rev. 113, 5989 (2013).

[19] M. E. Madjet, H. S. Chakraborty, and S. T. Manson, Phys. Rev. Lett. 99, 243003 (2007).
[20] R. Van Leeuwen and E. J. Baerends, Phys. Rev. A 49, 2421 (1994).

[21] B. Möbus, B. Magel, K. H. Schartner, B. Langer, U. Becker, M. Wildberger, and H. Schmoranzer, Phys. Rev. A 47, 3888 (1993).

[22] J. A. R. Samson and W. C. Stolte, J. Electron Spectrosc. Relat. Phenom. 123, 265 (2002).

[23] V. S. Yakovlev, J. Gagnon, N. Karpowicz, and F. Krausz, Phys. Rev. Lett. 105, 073001 (2010).

[24] E. P. Wigner, Phys. Rev. 98, 145 (1955).

[25] F. T. Smith, Phys. Rev. 118, 349 (1960).

[26] C. A. A. de Carvalho and H. M. Nussenzveig, Phys. Rep. 364, 83 (2002).

[27] J. M. Dahlström, D. Guénot, K. Klünder, M. Gisselbrecht, J. Mauritsson, A. L'Huillier, A. Maquet, and R. Taïeb, Chem. Phys. 414, 53 (2013).

[28] I. A. Ivanov and A. S. Kheifets, Phys. Rev. A 87, 063419 (2013).

[29] G. Onida, L. Reining, and A. Rubio, Rev. Mod. Phys. 74, 601 (2002).

[30] S. Petretti, Y. V. Vanne, A. Saenz, A. Castro, and P. Decleva, Phys. Rev. Lett. 104, 223001 (2010).

[31] J. Heslar, D. Telnov, and S.-I. Chu, Phys. Rev. A 83, 043414 (2011).

[32] J. P. Farrell, S. Petretti, J. Förster, B. K. McFarland, L. S. Spector, Y. V. Vanne, P. Decleva, P. H. Bucksbaum, A. Saenz, and M. Gühr, Phys. Rev. Lett. 107, 083001 (2011).

[33] D. Toffoil, and P. Decleva, J. Chem. Phys. 137, 134103 (2012).

[34] M. Hellgren, E. Rasanen, and E.K.U. Gross, arXiv:1309.0786.

[35] M.E. Madjet, T. Renger, D. E. Hopper, M. A. McCune, H. S. Chakraborty, Jan M. Rost, and S. T. Manson, Phys. Rev. A 81, 013202 (2010).

[36] S. W. J. Scully, E. D. Emmons, M.F. Gharaibeh, R. A. Phaneuf, A.L.D. Kilcoyne, A.S. Schlachter, S. Schippers, A. Müller, H.S. Chakraborty, M.E. Madjet, and J. M. Rost, Phys. Rev. Lett. 94, 065503 (2005).

[37] A. Rüdel, R. Hentges, U. Becker, H. S. Chakraborty, M. E. Madjet, and J.M. Rost, Phys. Rev. Lett. 89, 125503 (2002).

[38] H. S. Chakraborty, M. E. Madjet, T. Renger, Jan M. Rost, and S. T. Manson, Phys. Rev. A 79, 061201 (2009).

[39] M. Morscher, A.P. Seitsonen, S. Ito, H. Takagi, N. Dragoe, and T. Greber, Phys. Rev. A 82, 051201 (2010).

[40] U. Fano, Phys. Rev. 124, 1866 (1961).

[41] M. H. Javani, M. R. McCreary, A. B. Patel, M. E. Madjet, H. S. Chakraborty, and S. T. Manson, Eur. Phys. J. D 66, 189 (2012).

[42] V. Veniard, R. Taieb, and A. Maquet, Phys. Rev. Lett. 74, 4161 (1995).

[43] M. Chini, S. Gilbertson, S. D. Khan, and Z. Chang, Opt. Express 18, 13006 (2010). 